\title{
DEVELOPING A WEB BASED DECISION SUPPORT SYSTEM FOR DISASTER MANAGEMENT ON AILA AFFECTED AREA OF KOYRA UPAZILA
}

\author{
R. Ahasan ${ }^{1 *} \&$ S. A. Saimon ${ }^{2}$ \\ ${ }^{1}$ URP Discipline, Khulna University, Khulna, Bangladesh \\ ${ }^{2} J u n i o r$ Town Planner, Development Design Consultant (DDC), Khulna, Bangladesh \\ *Corresponding Author: rakibukahasan@gmail.com
}

\begin{abstract}
Bangladesh is one of the most disaster prone countries of the world and has been subjected to frequent natural disasters in many forms, particularly cyclonic storms and storm surges. The low-lying coastal areas are particularly vulnerable, thus placing these population, infrastructure, agriculture, livestock and economic development in a high-risk situation. The situation calls for an effective cyclone warning and dissemination system. With a view to address that this research intends to develop and manage a website containing all the information for helping in taking decision about disaster management. As its common knowledge that disaster victims need immediate, efficient and effective rescue and relief services, this system will provide relief and rescue workers with a platform easy to use, fast to search, retrieve and access information and most importantly will help in preventing the duplication of relief works. Overall, proper co-ordination will be ensured among the existing organization seems to be lacking due to the shortage of effective information dissemination system.
\end{abstract}

Keywords: Disaster management; warning and dissemination system; relief works

\section{INTRODUCTION}

Bangladesh is one of the most disaster-prone countries in the world. Physical hazards often cause considerable loss of life and catastrophic physical damage and disruption to society and the national economy, these include exceptional widespread of flooding, severe tropical cyclones and associated coastal storm surges, drought and earthquakes. The geographical setting of Bangladesh makes the country vulnerable to natural disasters. The mountains and hills bordering almost three-fourths of the country, along with the funnel shaped Bay of Bengal in the south, have made the country a meeting place of life-giving monsoon rains, but also make it subjected to the catastrophic ravages of natural disasters. The country has been subjected to frequent natural disasters in many forms, particularly cyclonic storms and storm surges. The low-lying coastal areas are particularly vulnerable, thus placing these population, infrastructure, agriculture, livestock and economic development in a high-risk situation. The situation calls for an effective cyclone warning and dissemination system. It's a fact that humans do not have the capacity to survive without food, water, medicine and shelter for more than a few days.

Since Bangladesh is a disaster prone country, it is subject to colossal damages to life and property almost every year. The effects of a natural disaster or a combination of more than one natural disaster may be direct loss of life and certainly damage to physical properties. Therefore, the consequences of these natural hazards and the resulting environmental degradation pose a serious threat to the economic development of the country. To overcome these issues and to be in a better position requires large amount of resources for disaster management including mitigation, recovery and preparedness. Therefore, an effective disaster warning and dissemination system is necessary. A timely and accurate alert system about impending disasters will help reduce the loss of life and property (Pramanik, 1991). Web based decision support system can be a useful tool by which dissemination of information will ensure accuracy and quickness. These mechanisms will help all the concerned people or organizations to have the adequate information about a disaster. Information dissemination system of Bangladesh is confined with traditional postal system, which takes lots of time. When a post disaster situation arises, the damage information of the certain disaster takes few days to reach to the center (DMB). 
Nevertheless, for effective disaster management it needs to be faster to save as many lives as possible. If the web based DSS can be introduced, then it can help by spreading the information of a disaster to everybody in real time. It can also help to pin pointing those locations which are most vulnerable. People can be saved by a huge number if this system can be adopted. Without the web based system the disaster management would be slow as before.

\section{CONCEPTUAL FRAMEWORK}

Disaster is an important issue now-a-days in Bangladesh. Every year Bangladesh experiences tremendous physical and economic losses due to natural disasters. These losses hamper the national development process grossly. Therefore, there must be an effective disaster management system to reduce the losses. To help the policy makers in formulating an effective disaster management plan, effective and efficient dissemination of data is much helpful. As a result, this step has been taken to develop a web based DSM for disaster management for a small community. Here an attempt has been taken to establish a DSM for disaster management for South-West Coastal Region of Khulna division, in a small context.

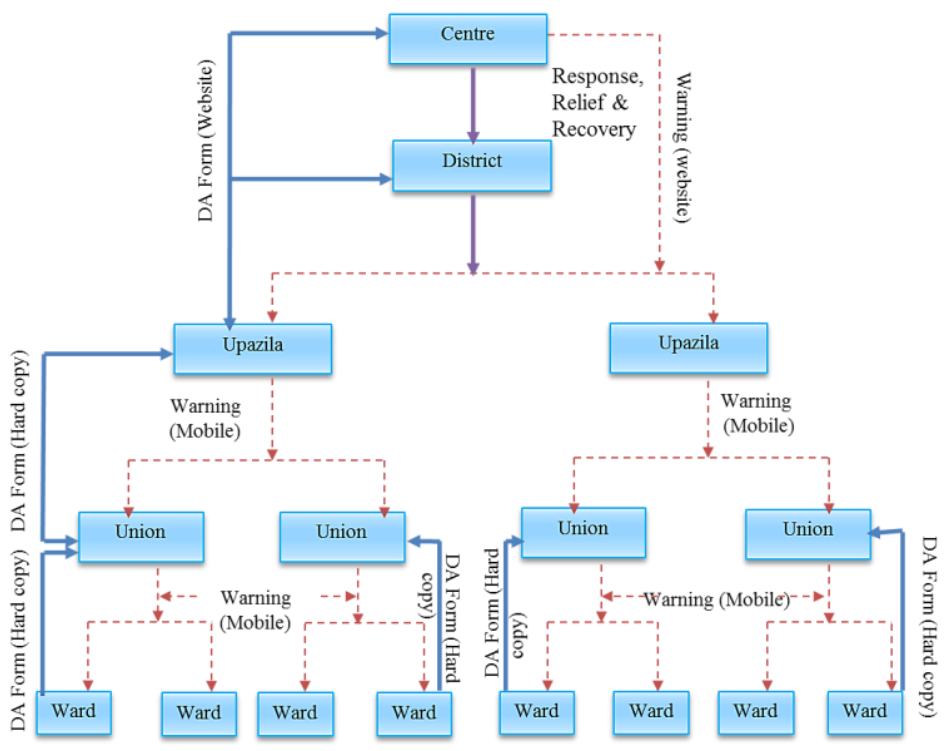

Fig. 1: Conceptual Framework

\section{NEED FOR EFFICIENT DISASTER RESPONSE SYSTEM}

The recurrent devastating disasters cause substantial losses in infrastructure, life and property in Bangladesh. Economic growth of the country significantly depends on efficient structural and non-structural measurement against disaster. As a poor country, Bangladesh cannot afford huge amounts of money required for structural measurement. That is why it is more rational to place emphasis on non-structural measures such as developing a website which will contain risk maps, damage information and relief situation etc. Ultimately, the web based procedure may help policy makers to make effective response against possible disasters in near future. Furthermore, these data will be stored in the website so that the data remain safe and all the people can use those data and maps. There are also many reasons behind developing a website. Those reasons are like disaster victims need immediate, efficient and effective rescue and relief services. That is why a website can be very handful in disseminating information. Also to provide relief and rescue workers with a platform easy to use, fast to search, retrieve and access information, prevent duplication of relief works. Overall, proper co-ordination among the existing organization seems to be lacking due to the shortage of effective information dissemination system.

\section{POSSIBLE SOLUTION}

The inevitability of natural disasters almost every years leads to the belief and concern that a system or some mechanism is required in order to mitigate the loss, or prevent the catastrophic events and aftermaths as well. That is why an effective early warning system is solely required which might have comprised of-

- Rapid damage assessment in the study area

- Mapping of routes for rescue and relief operation

- Information dissemination to the emergency service providers

- Provide capabilities for efficient data mining 


\section{WEBSITE CONTENT}

Before making of a website it is necessary to make content so that the data that will be required for each menu can be easily distributed. Content is most essential part before making a website because it helps to do the work in a planned way. The content of the website is divided in two parts. They are-

\section{WEBSITE}

- Main menu

- Sub Menu

In the main menu the tabs that will be kept are given below-

- HOME

- WARNING

- AREA \& MAP

- ACTIVE NGOs

- DAMAGE INFORMATION

- RECOVERY

The following content will be incorporated and included in the website.

- Risk map- Use as the primary layer to determine the areas where places are highly vulnerable.

- Road networkShortest path and alternate evacuation path delineation.

- Utility networkAssess the damage to communication network and water supply.

- School population dataAssess the vulnerable people present to

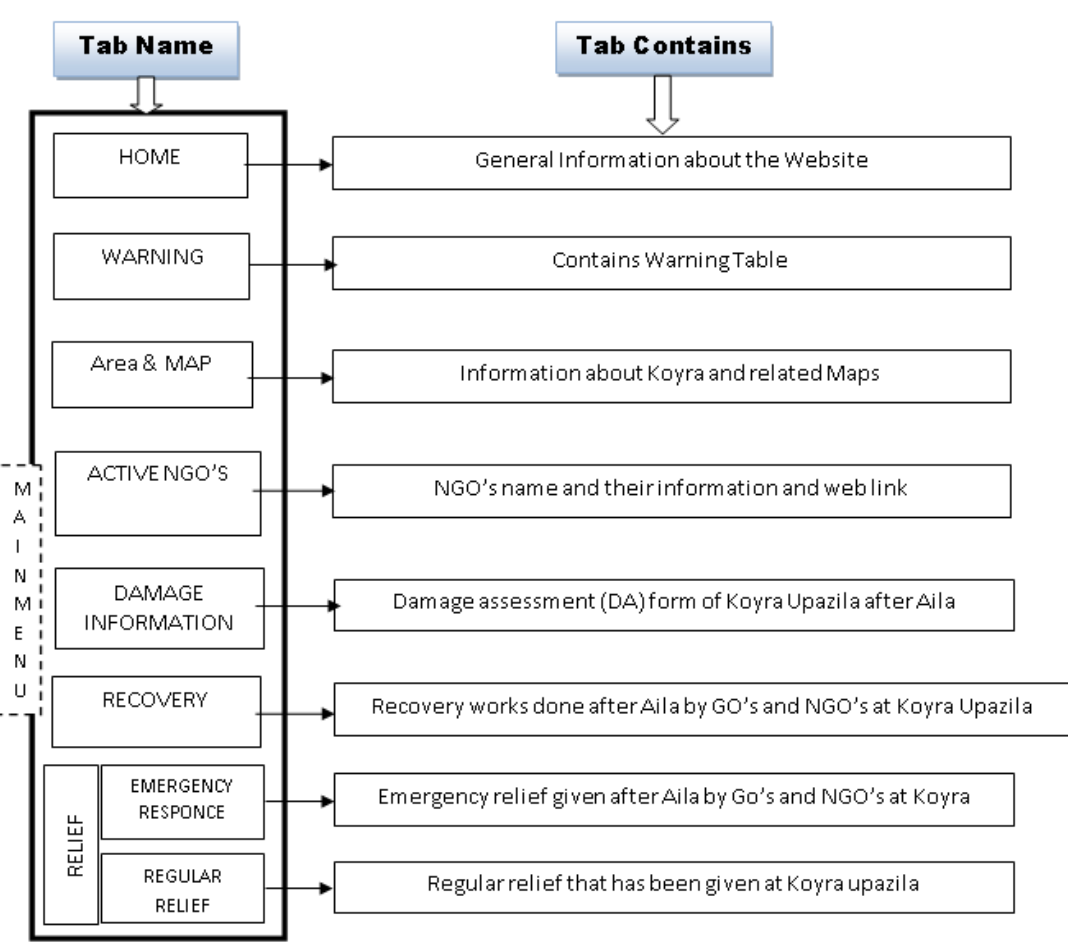
prioritize rescue operations.

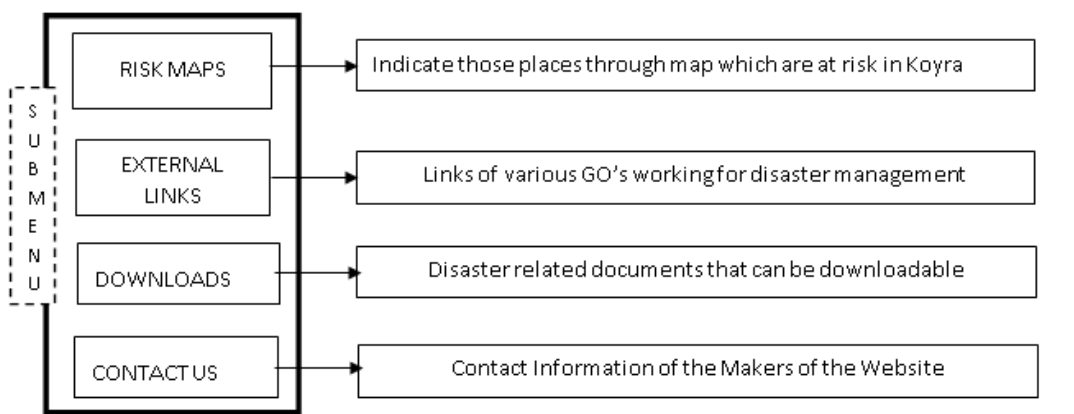

Fig. 2: Website Content

- Hospital and shelter data- Assess the capacity of the hospital and shelter locations in admission of disaster victims as well as act as destination point in network analysis.

\section{WHY IS SUCH SYSTEM USEFUL?}

The introduction of such website or early warning system will provide the following benefits-

- Proper and faster dissemination of information at the time of disaster.

- Better address the vast opportunities to utilize geo-data and tools via the WWW.

-Properly co-ordinates the activities and available resources among the existing bodies.

-Provides specialized / limited applications that meet specific needs.

-Platform to integrate information from other sources to best manage and rescue the disaster scenario.

- Relatively easy for user to use, search, retrieve and access through visualization.

\section{LONG-TERM RECOMMENDATIONS:}


- Work with communities, civil society, and government to bring basic services to scale (with a focus on disaster resilience).

- Develop local food storage system

- Introduce salinity tolerant varieties of rice

- Expand training on alternative livelihood and climate change adaptation

- Develop community food bank

- Strengthen micro-finance support

- WASH contingency plan and stock, including water/desalination plant

- Multipurpose disaster resilient market/school cum cyclone shelter

- Permanent family shelter and cluster houses for land less people

- Forestation/Watershed development

- Expand household and community water harvesting system

- Increase sanitation coverage in market and other public areas.

- Expand disaster resilient Watson system at household and community level

- Study on deep aquifer to explore safe water sources

- Incorporate DRR into the education program

Currently GOs and NGOs have been working to recover structural damages in the Aila affected zones. However, most of the embankments are still damaged and in some parts they are fully disappeared, where it is hardly possible to distinguish the locality from the water body. In every high tide water enters into locality through breaches and there is a risk of further inundation of the whole area in case of $5 \mathrm{~m}$ high tide. At the initial stage organizations were much more concerned with the emergency needs and responses. A number of GOs and NGOs provided food and non-food (cloths, wallet etc.) items to the affected families. Medical care, temporary shelter materials and wash kits were distributed by a numbers of local and international NGOs among the affected households. Local government officials at Padmopukur informed that they have already distributed 10.5 million BDT for rehabilitation works including $4000 \mathrm{VGF}$ cards. Each VGF card holder receives $20 \mathrm{~kg}$ rice per month.

\section{IMPORTANCE OF WEBSITE IN CONTEXT OF PLANNING}

The main consideration of this website is to help in taking decision about disaster management. The website will help in every aspect of the disaster management cycle. That are- prevention, mitigation, preparedness, response and recovery.

Prevention

- Current status of prevention.

- Adequate assessment and monitoring of disaster hazards and vulnerabilities.

- Risk maps.

Mitigation

- Continuous monitoring and evaluation.

- land use map.

- Assessing strength / capacity of the cyclone shelters.

- Identifying the soil salinity.

Preparedness

- Supporting coordination among different organizations (GOs \& NGOs)

- Helping to calculate the relief needed for the people under risk.

- Showing the signals and also giving information on the immediate response.

Recovery

- Type of recovery work is going and needed.

- The number of people benefited by the work.

- Which organizations are doing what type of recovery work.

Response

- First of all, the damage assessment form is provided in the website.

- It's helpful in calculating the need that is required in the affected area. 
- Response measures can be taken immediately prior to and following disaster based on the information in the website.

- This website will help to quantify the actual needs in the study area.

- For quickest dissemination of information's and decisions.

- The site can show the evacuation routes available in the study area.

- This site shows the works done or will be done or doing by various organizations for helping the people.

\section{INTENDED USERS OF THE WEBSITE}

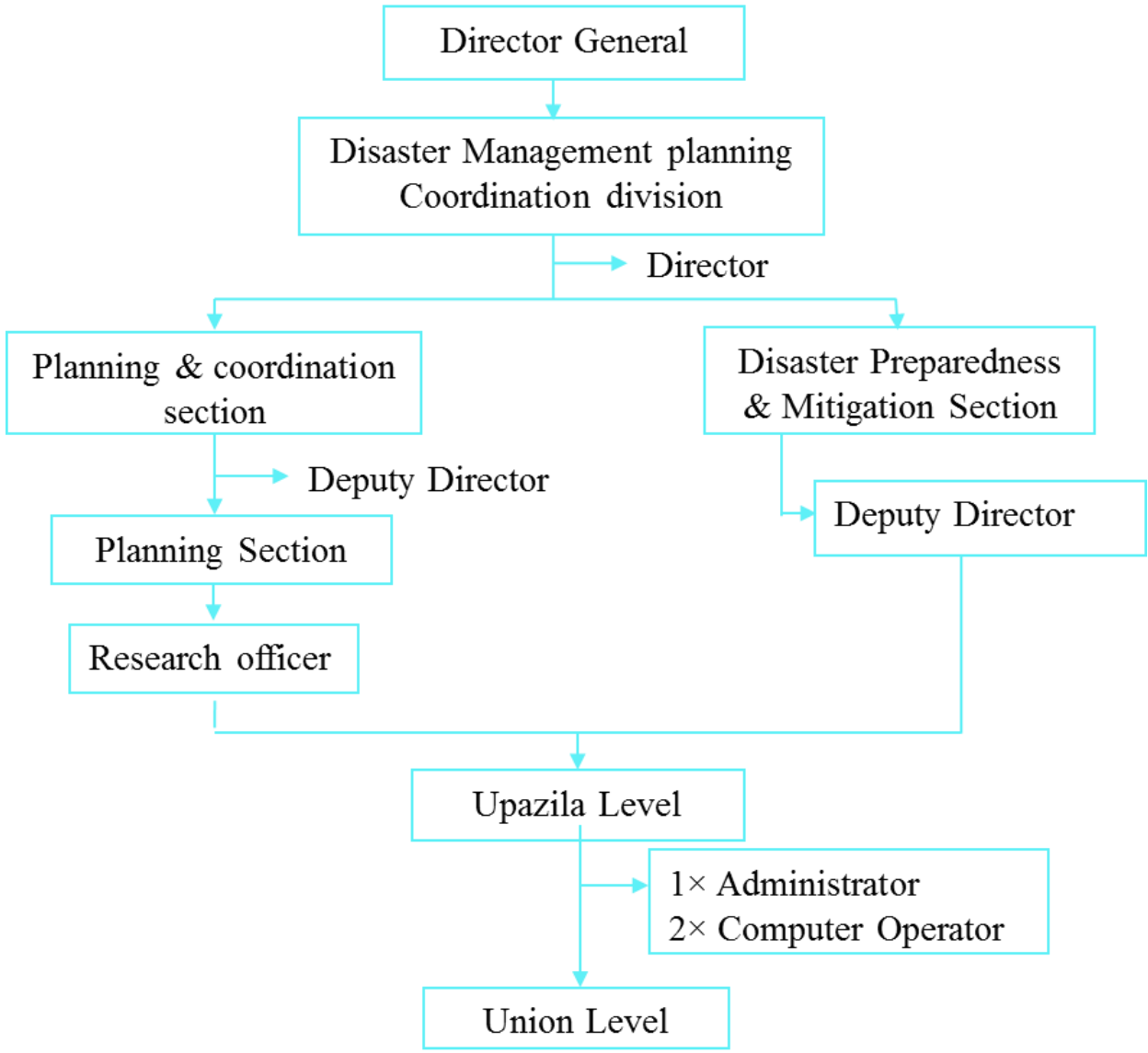

Fig. 3: Organogram of Disaster management Bureau who may use the website

\section{Government}

The government authorities can manage \& use the website for decision making. They can use this website to-

- Make decisions related to disaster management

- Disseminate warning quickly

- Managing a disaster

- An inventory of activities which could be open for all to see name of the agencies and officials, dissemination flow chart.

\section{NGO's \& Donor Agencies}

- NGOs can show their work \& support.

- An inventory of activities which could be open for all to see name of the agencies and officials, dissemination flow chart.

\section{People (From all over the World)}

- People, either living in this country or any foreign country, will get all updated news about a following disaster from this site.

- Various researchers can also be helped getting all the information from this site. 


\section{CONCLUDING REMARKS}

Cyclones are such a natural phenomenon for Bangladesh which recurs almost every year with a varied intensity. In this research a web based non-structure procedure has been followed to analyse and mitigate the damage of a probable disaster like cyclone. This web based system is also helpful in determining rapid damage assessment in the study area. Furthermore, it will help to disseminate information to the emergency service providers and also it provides capabilities for efficient data mining. It is the premise of this study that web based system can be a useful tool in for hazardidentification and analysis for implementing mitigation strategies. The current research is a step forward towards the development of a fully operational web based DSS for disaster management Koyra upazila for further adaptation and implementation by administrators. The disaster management approach for Koyra upazila consisting of prevention, reduction, mitigation, relief and rehabilitation has been prepared through the development of an accurate and comprehensive web base database. Web based DSS will provide required information and maps showing affected areas, road, lifeline structures, demographics and other baseline data to assist in search and rescue, relief, and rehabilitation in a timely manner. In addition, efficient management of the website is also discussed in this research. The web based disaster management system can be used as an integrated administrative and decision support system to respond to the immediate humanitarian and disaster relief operations pre, during and after the disaster. Bangladesh is a country of resource scarcity where structural measurements against disaster are very expensive and not always cost effective solutions. Therefore, non-structural measurement should be emphasized against floods or cyclones such as, well developed early warning system, forecasting system etc. Using web based potentiality, which can response effectively in pre, during and post disaster period. Taking cyclone as a common phenomenon for Bangladesh this type of non-structural measurement should be accentuated for better response.

\section{REFERENCES}

Ali, 1996. Vulnerability of Bangladesh to climate change and sea level rise through tropical cyclones and storm surges. Bangladesh Space Research and Remote Sensing Organization (SPARRSO), Agargaon, Sher-e-Bangla Nagar, Dhaka, Bangladesh.

Crossland, MD; Wynne, BE and Perkins, WC. 1995. Spatial decision support systems: An overview of technology and a test of efficacy. Decision Support Systems, 14: 219-235.

Dasgupta, S; Huq, M; Khan, ZH; Ahmed, MM; Mukherjee, N; Khan, MF and Pandey, K. 2010. Vulnerability of Bangladesh to Cyclones in a Changing Climate Potential Damages and Adaptation Cost. The World Bank Development Research Group Environment and Energy Team.

Disaster Management Bureau (DMB), 2009. Ministry of Disaster Management and Relief. Government of People's Republic of Bangladesh, Retrieved November 26, 2009, from http://www.dmb.gov.bd /situation_report.html

Government of the People's Republic of Bangladesh (2009). Bangladesh Climate Change Strategy and Action Plan, Dhaka.

Khalil, GM. 1992. Cyclones and Storm Surges in Bangladesh: Some Mitigative measures, Natural Hazards, 6:11-24.

Khan, RS. 1992. Cyclone hazard in Bangladesh. Community Development library, Dhaka, 86-102. National States Geographic Information Council. 2006. About NSGIC, Retrieved October 20, 2009, from http://www.nsgic.org/about/index.cfm

Radke, J; Cova, T; Sheridan, MF; Troy, A; Lan, M and Johnson, R. 2000. Application Challenges for Geographic Information Science: Implications for Research, Education, and Policy for Emergency Preparedness and Response. URISA Journal, 12(2): 15-30.

Rinner, C. 2003. Web-based Spatial Decision Support: Status and Research Directions. Journal of Geographic Information and Decision Analysis, 1(7): 14-31.

UNDP, 2005. Information Management for Natural Disasters: A Pilot Project for Kabul \& Kunduz Province, UNDP Project Report. 